\title{
Value of a new pathological classification of lumbar intervertebral disc herniation based on transforaminal endoscopic observations
}

\author{
LIN YANG and HONG-HUI LU
}

\author{
Department of Orthopedics, The Third Hospital of Beijing Armed Police Force, Beijing 100141, P.R. China
}

Received November 18, 2015; Accepted December 19, 2016

DOI: $10.3892 /$ etm.2017.4201

\begin{abstract}
Removal of herniated disc materials based on an imaging only method may not relieve symptoms in many patients. Therefore, the aim of the present study was to develop a transforaminal endoscopic method of classifying the pathological type of lumber intervertebral disc herniation and to compare the outcomes of surgery based on the pathological type with those of conventional endoscopic disc removal. The records of patients who received endoscopic transforaminal nucleotomy with foraminoplasty for symptomatic lumbar disc herniation between 2009 and 2013 were retrospectively reviewed. Patients were then divided into two groups: Group A, which consisted of 275 patients who received conventional endoscopic transforaminal nucleotomy with foraminoplasty between 2009 and 2011 and group B, which consisted of 316 patients who received 'targeted' endoscopic transforaminal nucleotomy with foraminoplasty between 2011 and 2013 (based on the pathological type of disc herniation identified at surgery, including fresh, calcified and scar type based on intraoperative observations). The results showed that there were no significant differences in age, gender, body mass index, symptom duration, operated segments or previous invasive therapies between the two groups. Moreover, evaluation of visual analogue scale pain scores and Oswestry disability index scores revealed that the patients in group B had a greater improvement in symptoms than those in group A $(\mathrm{P}<0.05)$. In addition, an age $>40$ years and a longer symptom duration were associated with the calcified type, and previous invasive therapy was associated with the scar type. Therefore, specific surgical treatment based on the transforaminal endoscopic pathological type can result in better outcomes for patients with lumbar disc herniation.
\end{abstract}

Correspondence to: Dr Hong-Hui Lu, Department of Orthopedics, The Third Hospital of Beijing Armed Police Force, 10 Xiaotun Road, Beijing 100141, P.R. China

E-mail: honghuilu5@sina.com

Key words: lumbar disc herniation, radiculopathy, transforaminal endoscopy, nucleotomy, foraminoplasty

\section{Introduction}

Endoscopic transforaminal nucleotomy with foraminoplasty is an accepted procedure for the treatment of lumbarintervertebral disc herniation, and is associated with substantially reduced surgical trauma compared to the usual dorsal approach (1). In addition, positive outcomes have previously been reported. This technique has the notable advantages of i) low invasive approach, ii) lower muscle manipulation iii) postoperative back pain reduction and fibrosis and iv) direct observation of decompressed root (2). A previous Swedish study also found that endoscopic transforaminal nucleotomy with foraminoplasty had a significant better result in visual analog scale (VAS) back and leg pain, walking distance and patient satisfaction (3). Kim et al (4) concluded that this may be effective surgical method in unilateral adjacent 2 levels lumbar disc herniation through 1 skin portal incision and Choi et al (5) also claimed that XMR-assisted endoscopic transforaminal nucleotomy with foraminoplasty is able to provide a precise skin entry site (5). Knight et al (6) also demonstrated that this intervention is able to improve symptoms and function that were still sustained 10 years later in a prospective study. Based on these positive outcomes (2-7), in January 2009, we began performing endoscopic transforaminal nucleotomy with foraminoplasty, and the observations indicated that lumbar disc herniations can be categorized into three pathological types, which are different than those described based on imaging analysis (8). While imaging analysis can identify the location of the herniation and help guide disc removal, it is believed that surgical treatment based on the three pathological types identified can result in improved surgical outcomes because surgery based on the pathological type better addresses the cause of the symptoms. In 2011 we began performing endoscopic transforaminal nucleotomy with foraminoplasty based on the three pathological types identified.

Thus, the aim of the present study was to describe the three specific pathological types among herniated discs and to determine whether a treatment tailored specifically to the pathological type present improves patient outcomes.

\section{Materials and methods}

Patients. The records of patients who received endoscopic transforaminal nucleotomy with foraminoplasty for symptomatic lumbar disc herniation between 2009 and 2013 at 
Table I. Patient demographic data.

\begin{tabular}{lrr}
\hline Characteristics & $\begin{array}{c}\text { Group A } \\
(\mathrm{n}=275)\end{array}$ & $\begin{array}{r}\text { Group B } \\
(\mathrm{n}=316)\end{array}$ \\
\hline Age (years) & $46.8 \pm 7.3$ & $50.2 \pm 8.6$ \\
Gender (female/male) & $147 / 128$ & $180 / 136$ \\
Body mass index & $22.4 \pm 3.1$ & $22.7 \pm 3.3$ \\
Symptom duration (n) & & \\
Acute pain within the past 2 months & 60 & 75 \\
Recurrence within the past 2 months & 75 & 85 \\
Longer than 2 months & 140 & 156 \\
Operated segments (n) & & \\
L2/3 & 7 & 10 \\
L3/4 & 13 & 16 \\
L4/5 & 123 & 133 \\
L5/6 (lumbarization of S1) & 5 & 8 \\
L5/S1 & 127 & 149 \\
Previous invasive therapy (n) & 70 & 81 \\
\hline
\end{tabular}

the Third Hospital of Beijing Armed Police Force (Beijing, China) were retrospectively reviewed. Patients were divided into two groups. Group A included patients who received conventional endoscopic transforaminal nucleotomy with foraminoplasty for removal of herniated disc material and received surgery between 2009 and 2011. Group B consisted of patients who received 'targeted' endoscopic transforaminal nucleotomy with foraminoplasty based on the pathological type identified at surgery. These patients received surgery between 2011 and 2013, and patient characteristics are presented in Table I.

Lumbar disc herniation was diagnosed based on symptoms and signs, laboratory examination, magnetic resonance imaging (MRI) and/or computed tomography (CT) data. The affected nerve root was determined according to the paraesthesia distribution, decreased muscle power and reduced tendon reflexes. Moreover, in order to be eligible for surgery patients should have failed a 4-6 week course of conventional conservative therapy. Patients with asymptomatic disc herniation were not eligible for surgery. If symptoms and signs were typical of disc herniation, surgery was performed even if the MRI or CT observations were negative because the herniated nucleus pulposus may return to the intervertebral space when a patient is placed in supine during MRI or CT examination. In addition, patients with a multilevel disease were excluded. The Institutional Review Board of the Third Hospital of Beijing Armed Police Force approved the present study, and because of its retrospective nature the requirement of patient informed consent was waived.

Surgical techniques. Surgeries were conducted with the transforaminal endoscopic surgical system (TESSYS) $(9,10)$, and conventional transforaminal endoscopic discectomy was performed as described by Schubert and Hoogland (2). The usual access for disease at L5/S1 and L4/5 was $12-14 \mathrm{~cm}$ lateral to the midline, and for $\mathrm{L} 3 / 4$ and $\mathrm{L} 2 / 3 \sim 10 \mathrm{~cm}$ lateral to the midline. Both nerve root and dorsal root ganglion are primarily located in the upper $1 / 3$ of the foramen. Bone was removed by drilling after reaching the superior articular process of the lower vertebra to expand the foramen when necessary. During drilling the end of the working cannula was placed in the midline on anteroposterior radiograph, while it was located in the posterosuperior margin of the lower vertebra on lateral radiograph. Following placement of the working channel, the nerve root was observed and nerve injury was avoided by direct manipulation. Extreme care was taken to avoid blood vessels in the foramen. A proper perfusion pressure was maintained, and a radiofrequency ablation system was used to provide hemostasis at the root of bleeding blood vessels.

In the conventional procedure, the herniated nucleus pulposus was removed through the working channel, and the annulus fibrous was not cut. If no nerve root was observed, the ligamentum flavum was identified and dissected using a radiofrequency probe to expose the nerve root. Since application of a ring saw for removing bone in the articular process may increase the incidence of nerve root injury, a spiral bone drill with a nerve protection device in front of the head was used. Representative preoperative and intraoperative images, surgical specimen and the surgical route are shown in Fig. 1.

In targeted therapy access to the canal was the same as in the conventional procedure. However, the ultimate surgical procedure was based on pathological observations identified at surgery. Pathological observations were categorized as fresh, calcified and scar type disc herniation. Fresh was characterized as annulus rupture with the nucleus pulposus protruding into the spinal canal compressing the dural sac and/or nerve root. In this case, the target of therapy was removing the herniated disc material. Calcified was characterized by calcification of herniated material that may or may not be compressing the dural sac and/or nerve root. Other pathological changes, including ossification of the posterior longitudinal ligament, hypertrophy of the ligamentum flavum, subchondral bone sclerosis and lateral recess stenosis may also be present. The calcified disc material, calcified posterior longitudinal ligament, thickened ligamentum flavum and subchondral bone scleroses are the targets. The volume of the spinal canal was expanded to restore the blood supply to the nerve root, and the nerve root was mobilized so that it moves smoothly when performing the straight leg raise test. An endoscopic drill bit, which has a relatively thin diameter, was used to remove the calcified material, which is different from the spiral drill bits used when placing the working channel to expand the foramen. The scar type was characterized as scar tissue inside the annulus fibrosus and/or surrounding the nerve root without the presence of herniated disc material. The goal of therapy was to remove the scar tissue such that the nerve root can move freely. A description of the three pathological classifications is shown in Fig. 2, the surgical goals are summarized in Fig. 3, and representative intraoperative images targeted therapy cases are shown in Fig. 4.

Follow-up and evaluation. All patients were followed-up every three months for a minimum of 18 months. Back and leg pain VAS (11) scores with 1 indicating minimal pain, and 10 indicating intense pain and Oswestry Disability Index (ODI) $(12,13)$ scores were determined preoperatively and at 
A

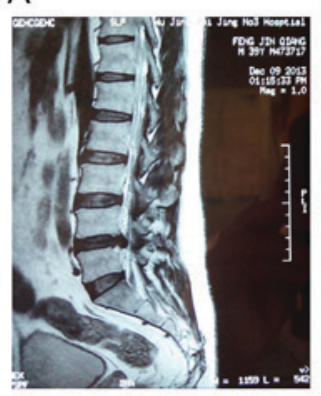

D

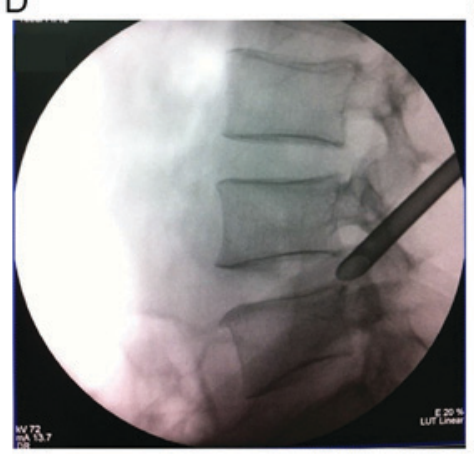

$\mathrm{F}$

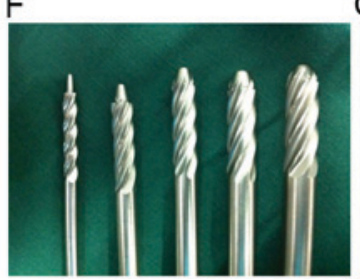

B

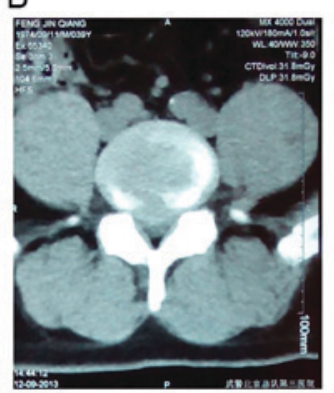

C

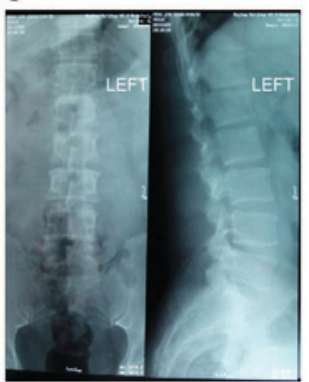

E
G

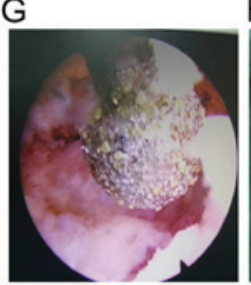

$\mathrm{H}$

Figure 1. Transforaminal endoscopic discectomy. Preoperative images of magnetic resonance imaging, computed tomography and X-ray were shown in (A-C), respectively. (D) Lateral radiograph showing surgical path entering the lower one-third of the foramen to avoid damaging both the nerve and vascular tissues; (E) intraoperative image showing there is ample space for the surgical tool such that the risk of damage to nerve or vessels is minimal; (F) spiral drill bits, (G) endoscopic drill bits and $(\mathrm{H})$ surgical specimens.

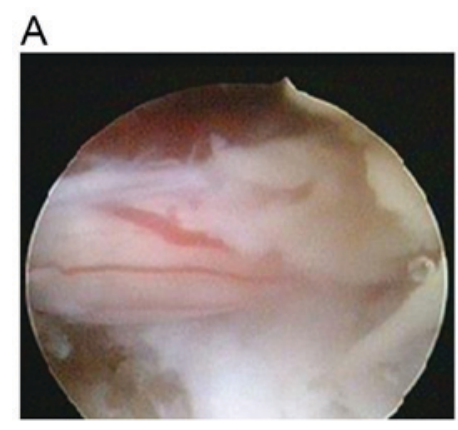

C

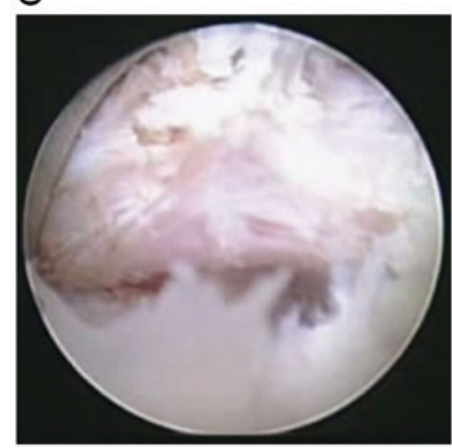

B

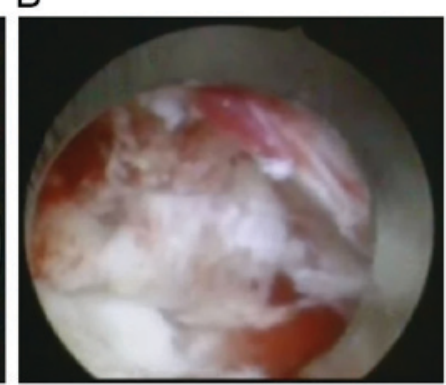

D

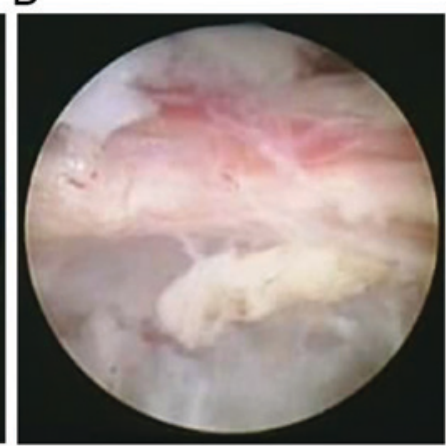

Figure 2. Transforaminal endoscopic pathological classifications of disc herniation. (A) The fresh type, where only disc herniation is observed and the nucleus pulposus and a rupture annulus fibrosus are noted without any secondary changes. (B) The calcified type, where long-standing disc herniation is present. Part of the disc has been absorbed and residual herniated disc is calcified. (C and D) The scar type, where scar tissue is observed on the surface of the nerve root. 


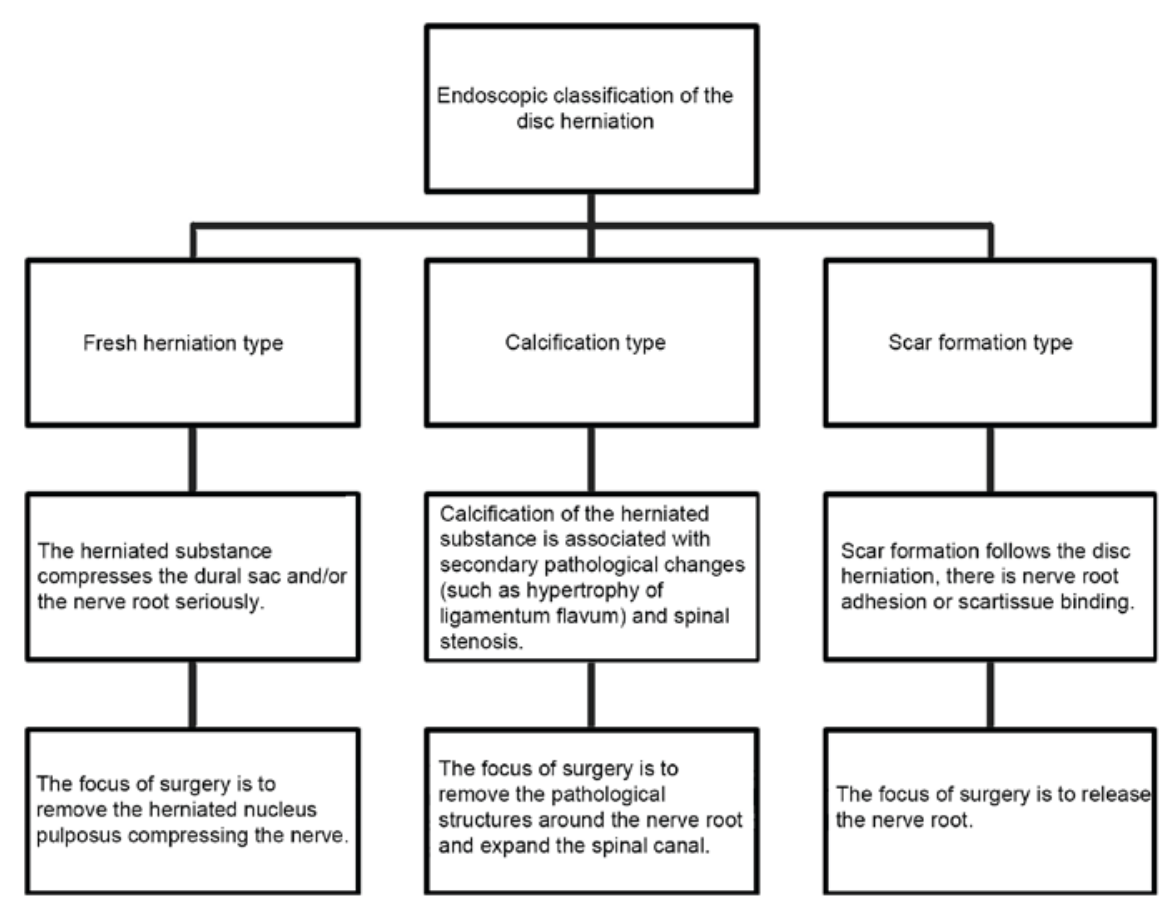

Figure 3. Pathological classifications of disc herniation and surgical goals.

each follow-up visit. The Macnab criteria (14) were also used to classify the outcomes.

Pathological classification and reliability. Video recordings were made of all operations and reviewed by a board-certified spine endoscopy surgeon for observation of the nucleus pulposus, rupture of the annulus fibrosus, spinal ligamentum flavum ossification, posterior longitudinal ligament calcification, scar tissue on the nerve root sheath surface, blood circulation of the nerve root and thecal sac, osteophyte in the posterior body edges of lumbar vertebrae, and lateral recess stenosis. Each case was classified into one of three pathological classifications: Fresh herniation, calcified and scar.

To determine the reliability of the pathological classifications, videos of 100 operations were selected randomly. Two examiners assessed each video, and assigned each video to one classification. For all video assessments, the examiner was masked regarding patient data. This process was repeated two weeks later by the same two examiners. Intra-rater and inter-rater reliability of video group assignment was determined by calculating a Kappa coefficient $(\kappa)$.

Histopathological staining. Surgical specimens were fixed in $10 \%$ formalin, embedded in paraffin, cut into $6-\mu \mathrm{m}$ slices, stained with hematoxylin-eosin and viewed under a light microscope (N-800M; Medical Instruments Co., Ltd., Jinan, China).

Statistical analysis. Data were presented by the mean \pm standard deviation for continuous variables, while the number and percentage were reported for categorical variables. To identify potential predictors of each type of disc herniation, three logistic regression models were performed using the forward stepwise method. The initial model included age, gender, body mass index (BMI), symptom duration, operated segment, previous invasive therapy and endoscopic pathological type. A value of $\mathrm{P}<0.10$ was applied to enter variables into the final model. Odds ratios (ORs) and 95\% confidence intervals (CIs) were also calculated. To examine the differences in change of surgical outcomes between two groups, Student's $t$-test was used for VAS pain and ODI scores, and $\chi^{2}$ test or Fisher's exact test was used for the Macnab criteria. All statistical analyses were conducted using SPSS software version 19.0 (IBM SPSS, Armonk, NY, USA). $\mathrm{P}<0.05$ was used to indicate a statistically significant difference.

\section{Results}

Participants. A total of 591 patients (327 females and 264 males) with a mean age of 48.45 years were included. Of the 591 patients, $135(22.8 \%)$ had acute pain (i.e., first episode of back/leg pain within the past two months), 160 (27.1\%) had a recurrence of previous symptoms within the past two months, and $296(50.1 \%)$ reported symptoms of longer than two months. There were 275 patients in group A and 316 in group B. Moreover, there were no significant differences in age, gender, BMI, symptom duration, operated segments or previous invasive therapy between the two groups. The patient demographic data are summarized in Table I.

Surgery was only performed for patients who were definitely diagnosed with lumbar disc herniation by symptoms or imaging findings and did not respond to the regular conservative treatment. This population accounted for only a small portion $(\sim 10 \%)$ of those diagnosed with lumbar disc herniation during the study period, and an operation was performed on only one segment for every patient. In the majority, but not all patients, the annulus was ruptured. A total of 17 patients were operated at segment L2/3, 29 at L3/4, 256 at L4/5, 13 at L5/6 (lumbarization of S1) and 276 at L5/S1. Among the 591 patients, 151 (25.5\%) had undergone previous invasive 

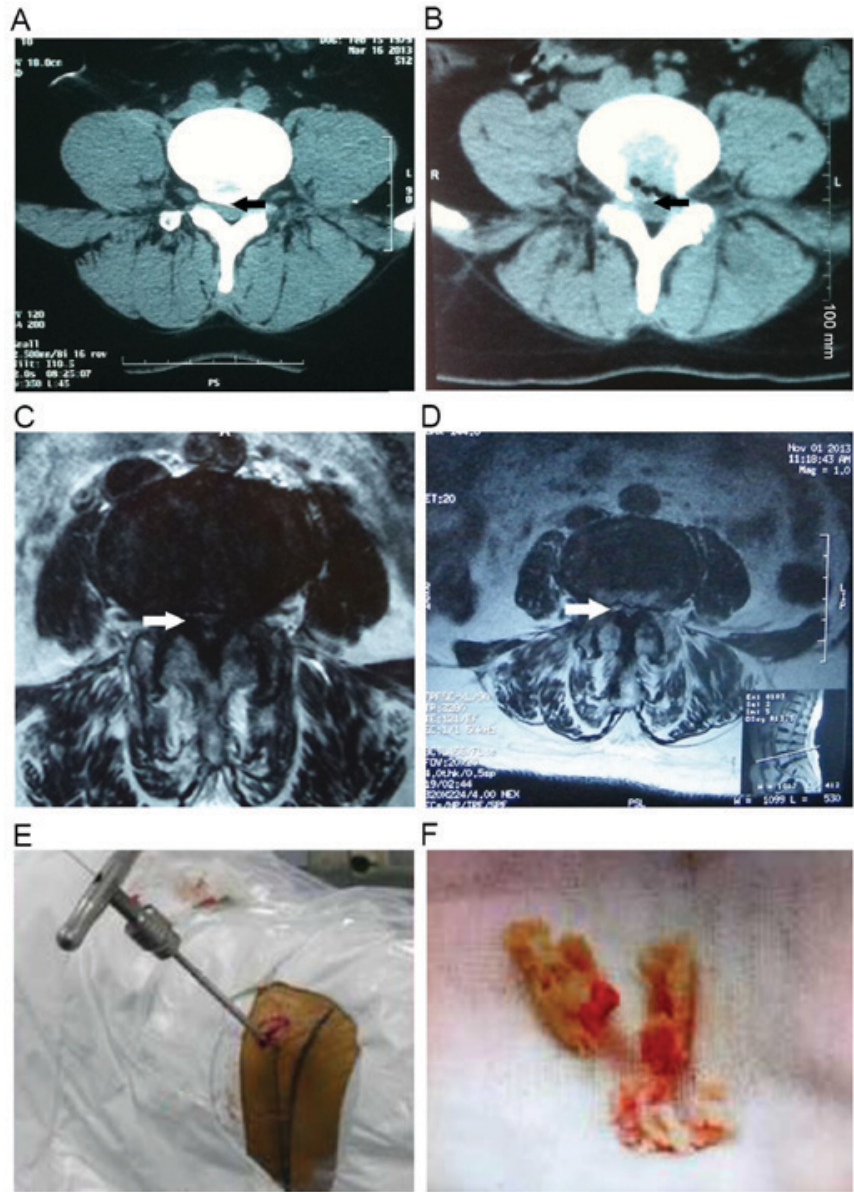

Figure 4. Representative images from 2 cases of targeted therapy. Case 1: (A) Preoperative CT showed calcification of a herniated disc (black arrow). (B) Postoperative CT verified that the calcifications were removed (black arrow). Case 2: (C) Preoperative MRI revealed ligamentum flavum hypertrophy and ossification (white arrow), (D) postoperative MRI showed that the calcifications were removed (white arrow) and in (E and F) the ligamentum flavum was removed. CT, computed tomography; MRI, magnetic resonance imaging.

therapies, including open surgery and percutaneous ablative techniques on the same segment.

Pathological classifications. Review of the operative videos revealed that there were 186 cases of fresh type, 313 of calcified type and 92 of scar type. Representative histopathological images of the three types are shown in Fig. 5. In the fresh type, the nucleus pulposus had shrunk, the stroma had decreased and increased numbers of disordered collagen fibers and cell necrosis were noted. In the calcified type, red staining calcifications in the ruptured annulus fibrosus were noted, and chondrocytes could seldomly be observed. In the scar type, proliferation of capillaries and inflammatory cell infiltration in tissue removed from the surface of the nerve sheath was noted. The agreement between histological and surgical observations for fresh, calcified and scar type specimens were 92.3, 96.8 and $72.4 \%$, respectively.

Reliability of pathological classifications and associations with patient characteristics. The intra-rater reliability Kappa coefficient for classifying patients into one of the three types was 0.82 for examiner 1 and 0.78 for examiner 2 . The inter-rater reliability Kappa coefficient for the classification was 0.76 for the first comparison and 0.77 for the second comparison (Table II). In addition, the results of the multiple logistic regression analysis are summarized in Table III. The most predictive observation of the calcified type was an age $>40$ years $(\mathrm{OR}=2.32$; 95\% CI: 1.31-4.02, $\mathrm{P}=0.002)$, followed by a longer symptom duration $(\mathrm{OR}=2.17$; 95\% CI: $1.05-3.54$, $\mathrm{P}=0.015)$. Moreover, previous invasive therapy was most predictive of the scar type $(\mathrm{OR}=2.98 ; 95 \% \mathrm{CI}: 1.43-4.68$, $\mathrm{P}=0.008)$.

Clinical outcomes. Evaluation of VAS pain and ODI scores revealed that patients in group B had a greater improvement in symptoms than those in group A (all, $\mathrm{P}<0.05$ ) (Tables IV and V). The outcomes of the two groups evaluated by the Macnab criteria were not significantly different $(\mathrm{P}=0.563$; Table V). In both groups, some cases of transitory dysaesthesia or hyperalgesia were reported $(<10 \%)$, which were resolved within 2-4 weeks of treatment with oral corticoids in all cases. Furthermore, no dural tears, neurological damage or wound infections occurred in either group.

The recurrence rate was $1.0 \%(3 / 275)$ in group A and $0.3 \%$ $(1 / 316)$ in group B. In group A, the three patients with recurrence received a second surgery, of which two were identified as the calcified type and one as the scar type. Patients in group A received conventional endoscopic transforaminal nucleotomy with foraminoplasty, and surgery aimed to remove the nucleus pulposus, however, the true lesions causing symptoms 
A

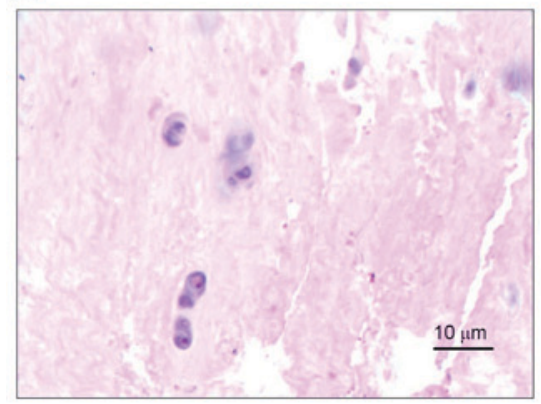

C

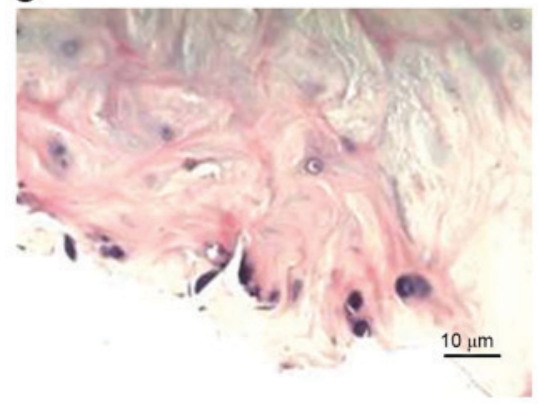

B

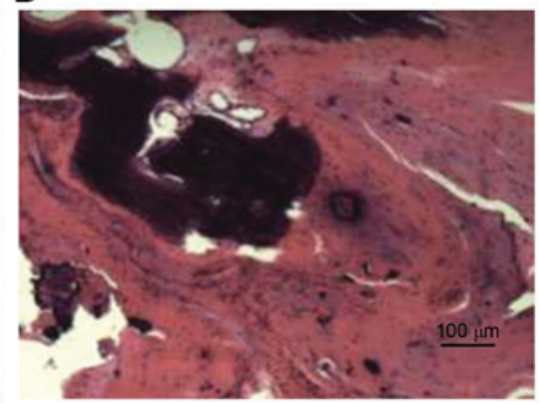

D

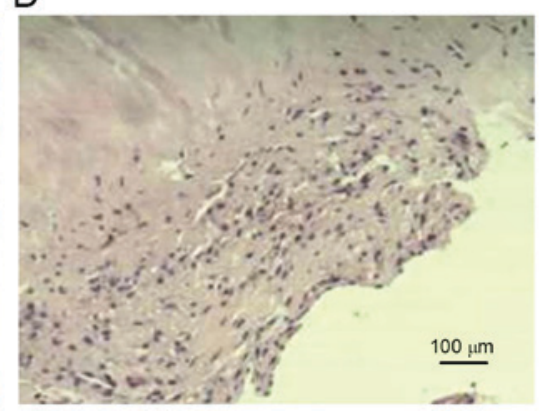

Figure 5. Histopathological examination of surgical specimens from different types of disc herniation. (A) The fresh type, where stroma is decreased, and increased disordered collagen fibers are observed. (B and C) The calcified type, where red staining calcifications in the ruptured annulus fibrosus and chondrocyte necrosis are observed. (D) The scar type, where disordered collagen fibers, proliferation of capillaries and inflammatory cell infiltration are observed. (A and C, magnification x400; B and D, magnification, $\mathrm{x} 100$ ).

Table II. Reliability of the transforaminal endoscopic pathological classification.

\begin{tabular}{lll}
\hline Variable & Kappa (SE) & $95 \%$ CI \\
\hline Intra-rater reliability & & \\
Examiner 1 & $0.82(0.04)$ & $0.72-0.92$ \\
Examiner 2 & $0.78(0.05)$ & $0.69-0.87$ \\
Inter-rater reliability & & \\
Comparison 1 & $0.76(0.06)$ & $0.67-0.85$ \\
Comparison 2 & $0.77(0.05)$ & $0.69-0.85$ \\
\hline
\end{tabular}

SE, standard error; CI, confidence interval.

were not removed. In group B, the scar type was found in the one patient with a recurrence. This patient had scar type, and the nerve root adhesions were difficult to release resulting in a poor outcome.

\section{Discussion}

The present study has presented a new pathological classification of lumbar disc herniation based on endoscopic observations. In total, three pathological types were identified and referred to as fresh, calcified and scar, and definitive treatment based on the type was associated with improved outcomes when compared to conventional removal of the protruding nucleus pulposus only. The reliability of identification of the three pathological types was good, and the types were consistent with histopathological examination of specimens removed at surgery.
Since its introduction, endoscopic transforaminal nucleotomy with foraminoplasty has become a widely accepted method of treating lumbar disc disease that is associated with good surgical outcomes and minimal surgical trauma (4-6). Novel approaches to the procedure include magnetic resonance assisted surgery (5), irrigation discectomy (15), and an 'inside out' technique that identifies pain generators with the patient in an awake and aware state using local anesthesia (16). Although the procedure is generally considered safe, reported complications include post-discectomy pseudocyst formation (17), dural tears (18) and exiting root injury (19). While some studies have indicated that the rate of recurrence is higher with endoscopic procedures, surgical indications and surgeon experience are crucial factors that affect outcomes (7). In fact, a study by Genevay et al (20) identified wide variability in the number and type of eligibility criteria of back pain syndromes and surgical indications.

The natural history of lumbar disc herniation is complex and variable, and degeneration of the disc continues two years after onset. It is generally recommended that six to eight weeks of conservative treatment should be performed, and surgical treatment should be carried out if conservative treatment fails (21). Masui et al (22) followed 21 patients with lumbar disc herniation treated non-surgically with serial MRI for a minimum of seven years. The mean space occupying the ratio of herniation demonstrated a significant reduction both on the two-year and final scans, and progression of degeneration of the intervertebral disc was observed in all patients on the final scan. Furthermore, no MRI factors were detected which could distinguish patients who were and were not eventually to develop lumbago and/or sciatica, and morphological changes of lumbar disc herniation continued 
Table III. Multiple logistic regression analysis.

\begin{tabular}{llcr}
\hline Disc herniation classification & \multicolumn{1}{c}{ Predictors } & Odds ratio & 95\% CI \\
\hline Fresh & Age $<40$ years & 1.52 & $1.03-2.74$ \\
& Symptoms $<2$ months & 1.65 & $1.06-2.86$ \\
Calcified & Age $>$ 40 years & 2.32 & $1.31-4.02$ \\
& Symptoms $>2$ months & 2.17 & 0.043 \\
Scar & Symptoms $>2$ months & 1.95 & $1.05-3.54$ \\
& Previous invasive therapy & 2.98 & 0.002 \\
& & & 0.015 \\
\hline
\end{tabular}

CI, confidence interval.

Table IV. Comparisons of changes in the visual analogue scale for pain and Oswestry disability index between groups.

\begin{tabular}{|c|c|c|c|c|c|c|}
\hline \multirow[b]{2}{*}{ Variable } & \multicolumn{3}{|c|}{ Group A } & \multicolumn{3}{|c|}{ Group B } \\
\hline & Pre & Post & Difference & Pre & Post & Difference \\
\hline \multicolumn{7}{|c|}{ Visual analogue scale for pain } \\
\hline Back pain & 8.5 & 3.1 & 5.4 & 8.7 & 1.3 & $7.4^{\mathrm{a}}$ \\
\hline Leg pain & 8.8 & 2.8 & 6.0 & 8.6 & 1.2 & $7.4^{\mathrm{a}}$ \\
\hline Oswestry disability index & 28.5 & 13.1 & 15.4 & 30.1 & 8.3 & $21.8^{\mathrm{a}}$ \\
\hline
\end{tabular}

${ }^{a}$ Compared with group A, the change of score of group B is significantly greater $(\mathrm{P}<0.05)$.

Table V. MacNab classification.

\begin{tabular}{lccc}
\hline Grade & Group A & Group B $^{\mathrm{a}}$ & Total \\
\hline Excellent & $137(49.8)$ & $166(52.5)$ & $303(51.3)$ \\
Good & $122(44.4)$ & $138(43.7)$ & $260(44.0)$ \\
Fair & $13(4.7)$ & $11(3.5)$ & $24(4.1)$ \\
Poor & $3(1.0)$ & $1(0.3)$ & $4(0.7)$ \\
\hline
\end{tabular}

Data are presented as $n(\%) .{ }^{\mathrm{a}} \mathrm{P}=0.563$ according to Fisher's exact test.

to occur even after two years. Parisini et al (23) examined the results of 129 patients who received surgery for lumbar disc herniation and reported excellent or good short-term results in $95 \%$ of cases, which decreased to $87 \%$ at a follow-up of 12.4 years. Atlas et al (24) performed a 10-year long-term follow-up study for 400 patients with sciatica caused by lumbar disc herniation, of which 217 received surgery and 183 conservative treatments. At the last follow-up, $69 \%$ of patients who received surgery considered that surgery improved their symptoms and $71 \%$ of patients were satisfied with the status quo; the rates were 61 and $56 \%$, respectively, in patients who received conservative treatment.

Endoscopic transforaminal nucleotomy with foraminoplasty for lumbar disk herniation not only has evident therapeutic advantages $(1,10)$, but the enhanced visualization can provide new insights into the disease process. Understanding and treatment of lumbar disc herniation has focused on removal of herniated disc material. However, secondary changes such as ligamentum flavum ossification, posterior longitudinal ligament calcification, poor blood circulation of the nerve root and thecal sac and stenosis of the lateral recess are often identified. Usually, excision of the ligamentum flavum or enlargement of the neural canal is performed, but in a large percentage of cases symptoms are not relieved.

In the present study, lumbar disc herniation observed under endoscopy was classified into three types: Fresh herniation, calcified and scar. In the fresh herniation, after annulus rupture the nucleus pulposus protruding into the spinal canal may compress the dural sac and/or nerve root resulting in low and leg pain. In the calcified type the extruded nucleus is absorbed spontaneously, and the symptom may diminish or completely resolve. However, in certain cases the extruded material may become calcified, particularly in elderly patients with a prolonged disease course (10). Other pathological changes, including ossification of the posterior longitudinal ligament, hypertrophy of the ligamentum flavum, subchondral bone sclerosis and lateral recess stenosis may also be present $(10,25)$. Altogether, these factors can result in a reduced spinal canal volume and the calcified tissue may compress or stimulate the dural sac and/or the nerve root. Scar type is associated with scar tissue around the nerve, and may be the result of an immune reaction. Under normal circumstances, the nucleus pulposus is contained within the annulus fibrosus and isolated from the immune system. Following herniation, it becomes a foreign body and may induce an immune response (26). This may be the reason for spontaneous absorption or calcification of the herniated nucleus pulposus observed in some cases (27). 
However, the immune response is also an inflammatory process, and can result in scar tissue formation. If the scar tissue is limited to the inside the annulus fibrosus, pink fibrous tissue may be observed at endoscopy and symptoms may be completely relieved after removing it. Moreover, if the scar tissue is adhered to the surface of the nerve root, radicular pain may not be completely relieved even if the nerve root is carefully dissected. In these cases no obvious herniated material can be observed at endoscopy, and the dural sac and/or nerve root may not be compressed (28). In addition, the present study has identified that these three types are not necessarily associated with each other. For example, in some elderly patients with a prolonged disease course a protruding nucleus pulposus compressing the dural sac and/or nerve root is identified but no calcifications are observed. This would be considered a fresh herniation type.

It is hypothesized by the present authors that the three pathological types may represent stages in the progression of the disease. Fresh type represents the early stage of the disease when herniation first occurs, and calcified and scar type are later phases but have different outcomes. Moreover, disc herniation as a result of degeneration and/or trauma can result in rupture of the annulus fibrosus and extrusion of the nucleus pulposus, which compresses the nerve root and thecal sac (25). However, the tissue is also exposed to the immune system. An immune response may be a partial cause of radicular symptoms (26), and may eventually lead to calcification of the extruded tissue or the formation of scar tissue. This postulation is somewhat supported by the observation that the calcified type was associated with a more advanced age and longer symptom duration, and scar type was associated with previous invasive therapy. Most importantly, targeted treatment based on the endoscopic observations resulted in better outcomes as measured by VAS pain and ODI scores compared to conventional management of simply removing the herniated disc material. In addition, it should be noted that there was no difference in MacNab score between the two groups. A VAS pain score is a measure of subjective feelings of pain, and the ODI is also a measure of pain and primarily the ability to perform activities of daily life. MacNab criteria are primarily based on whether pain affects the ability to work.

Although symptoms such as pain were not significantly improved following surgery, the changes in the VAS score and ODI were not evident in several patients of group A. However, those patients in group A were able to return to work and were graded as 'good'. Based on the aforementioned explanations, some patients in group A did not have a 'fair or poor' MacNab grade, although the therapeutic efficacy of surgery for those patients was poor. That is the possible reason that the MacNab grade was comparable between groups A and B. However, the changes between post- and pre-operative in VAS and ODI scores in group B were more significantly observed than in group A.

In the present study, there was relatively lower agreement between histological observations and clinical intraoperative assessment in scar type herniation compared to the other types. Moreover, sample collection was relatively difficult in scar type herniation. In the collected samples, there were scattered adhesions on the surface of the nerve roots, which did not meet the requirement for pathological staining. In the collected annulus fibrosus, contamination of scar tissues on the surface with cells from the annulus fibrosus could not be avoided, and these cells were hard to differentiate. However, only a pathological examination could differentiate these cells from scar tissues. This may be the reason for the agreement between histological observations and clinical intraoperative assessment with the scar type herniation.

There are a number of limitations to the present study. Firstly, there was lack of a systematic procedure for the diagnosis of the pain generator. Secondly, patients were not classified by imaging data (e.g., the McCulloch classification) for comparison with the three pathological types. Classification of lumbar disc herniation according to preoperative imaging data can only indicate the location of the herniation, and in many cases is not predictive of a surgical outcome. Thus, we do not believe it would be useful to strictly compare imaging data with the three pathological types.

In conclusion, the present study suggests three distinct pathological types of lumber disc herniation, and targeted surgical therapy based on the endoscopic pathological type. This resulted in better outcomes than simple removal of herniated disc material. These results suggest that the removal of the herniated material should not be the sole goal of surgery, and that factors other than disc herniation alone can be responsible for pain associated with lumbar disc herniation.

\section{References}

1. Gibson JN, Cowie JG and Iprenburg M: Transforaminal endoscopic spinal surgery: The future 'gold standard' for discectomy?-A review. Surgeon 10: 290-296, 2012.

2. Schubert $M$ and Hoogland T: Endoscopic transforaminal nucleotomy with foraminoplasty for lumbar disk herniation. Oper Orthop Traumatol 17: 641-661, 2005 (In English, German).

3. Iprenburg M and Godschalx A: Transforaminal endoscopic surgery in lumbar disc herniation in an economic crisis-the TESSYS method. US Musculoskeletal Review 3: 47-49, 2008.

4. Kim HS, Ju CI, Kim SW, Kim JG, Lee SM and Kim BW: Minimally invasive percutaneous endoscopic 2 levels adjacent lumbar discectomy through 1 portal skin incision: Preliminary study. Asian J Neurosurg 10: 95-101, 2015.

5. Choi G, Modi HN, Prada N, Ahn TJ, Myung SH, Gang MS and Lee SH: Clinical results of XMR-assisted percutaneous transforaminal endoscopic lumbar discectomy. J Orthop Surg Res 8: 14, 2013.

6. Knight MT, Jago I, Norris C, Midwinter L and Boynes C: Transforaminal endoscopic lumbar decompression \& foraminoplasty: A 10 year prospective survivability outcome study of the treatment of foraminal stenosis and failed back surgery. Int $\mathbf{J}$ Spine Surg 8: 21, 2014.

7. Anichini G, Landi A, Caporlingua F, Beer-Furlan A, Brogna C, Delfini R and Passacantilli E: Lumbar endoscopic microdiscectomy: Where are we now? An updated literature review focused on clinical outcome, complications and rate of recurrence. Biomed Res Int 2015: 417801, 2015.

8. Milette PC: Classification, diagnostic imaging, and imaging characterization of a lumbar herniated disk. Radiol Clin North Am 38: 1267-1292, 2000.

9. TESSYS technique (transforaminal endoscopic surgical system). Available at: http://www.joimax.com/us/products/endoscopy/tessys/TESSYS_concept.php. Accessed Jan 2, 2015

10. Nellensteijn J, Ostelo R, Bartels R, Peul W, van Royen B and van Tulder M: Transforaminal endoscopic surgery for symptomatic lumbar disc herniations: A systematic review of the literature. Eur Spine J 19: 181-204, 2010.

11. Aitken RC: Measurement of feelings using visual analogue scales. Proc R Soc Med 62: 989-993, 1969.

12. Fairbank JC, Couper J, Davies JB and O'Brien JP: The Oswestry low back pain disability questionnaire. Physiotherapy 66: 271-273, 1980. 
13. Monticone M, Baiardi P, Ferrari S, Foti C, Mugnai R, Pillastrini P, Vanti $C$ and Zanoli G: Development of the Italian version of the Oswestry Disability Index (ODI-I): A cross-cultural adaptation, reliability, and validity study. Spine (Phila $\mathrm{Pa} 1976) 34$ : 2090-2095, 2009.

14. Macnab I: Negative disc exploration. An analysis of the causes of nerve-root involvement in sixty-eight patients. J Bone Joint Surg Am 53: 891-903, 1971.

15. Soliman HM: Irrigation endoscopic discectomy: A novel percutaneous approach for lumbar disc prolapse. Eur Spine J 22 1037-1044, 2013.

16. Gore S and Yeung A: The 'inside out' transforaminal technique to treat lumbar spinal pain in an awake and aware patient under local anesthesia: Results and a review of the literature. Int J Spine Surg 8, 2014

17. Kang SH and Park SW: Symptomatic post-discectomy pseudocyst after endoscopic lumbar discectomy. J Korean Neurosurg Soc 49: 31-36, 2011

18. Ahn Y, Lee HY, Lee SH and Lee JH: Dural tears in percutaneous endoscopic lumbar discectomy. Eur Spine J 20: 58-64, 2011.

19. Choi I, Ahn JO, So WS, Lee SJ, Choi IJ and Kim H: Exiting root injury in transforaminal endoscopic discectomy: Preoperative image considerations for safety. Eur Spine J 22: 2481-2487, 2013.

20. Genevay S, Atlas SJ and Katz JN: Variation in eligibility criteria from studies of radiculopathy due to a herniated disc and of neurogenic claudication due to lumbar spinal stenosis: A structured literature review. Spine (Phila Pa 1976) 35: 803-811, 2010.

21. Awad JN and Moskovich R: Lumbar disc herniations: Surgical versus nonsurgical treatment. Clin Orthop Relat Res 443: 183-197, 2006.

22. Masui T, Yukawa Y, Nakamura S, Kajino G, Matsubara Y, Kato $\mathrm{F}$ and Ishiguro N: Natural history of patients with lumbar disc herniation observed by magnetic resonance imaging for minimum 7 years. J Spinal Disord Tech 18: 121-126, 2005.
23. Parisini P, Di Silvestre M, Greggi T, Miglietta A and Paderni S: Lumbar disc excision in children and adolescents. Spine (Phila Pa 1976) 26: 1997-2000, 2001.

24. Atlas SJ, Keller RB, Wu YA, Deyo RA and Singer DE: Long-term outcomes of surgical and nonsurgical management of sciatica secondary to a lumbar disc herniation: 10 year results from the Maine lumbar spine study. Spine (Phila Pa 1976) 30: 927-935, 2005.

25. Yoshiiwa T, Miyazaki M, Kawano M, Ikeda S and Tsumura H: Analysis of the relationship between hypertrophy of the ligamentum flavum and lumbar segmental motion with aging process. Asian Spine J 10: 528-535, 2016.

26. Hasegawa T, An HS, Inufusa A, Mikawa Y and Watanabe R: The effect of age on inflammatory responses and nerve root injuries after lumbar disc herniation: An experimental study in a canine model. Spine (Phila Pa 1976) 25: 937-940, 2000.

27. Shamji MF, Allen KD, So S, Jing L, Adams SB Jr, Schuh R, Huebner J, Kraus VB, Friedman AH, Setton LA and Richardson WJ: Gait abnormalities and inflammatory cytokines in an autologous nucleus pulposus model of radiculopathy. Spine (Phila Pa 1976) 34: 648-654, 2009.

28. Wassenaar M, van Rijn RM, van Tulder MW, Verhagen AP, van der Windt DA, Koes BW, de Boer MR, Ginai AZ and Ostelo RW: Magnetic resonance imaging for diagnosing lumbar spinal pathology in adult patients with low back pain or sciatica: A diagnostic systematic review. Eur Spine J 21: 220-227, 2012. 